\title{
The United Kingdom 2009 Swine Flu Outbreak As Recorded in Real Time by General Practitioners
}

\author{
Hershel Jick, ${ }^{1}$ Dean S. MacLaughlin,, ${ }^{1}$ Pascal Egger, ${ }^{2}$ and Peter Wiggins ${ }^{3}$ \\ ${ }^{1}$ Boston Collaborative Drug Surveillance Program, Boston University School of Medicine, 11 Muzzey Street, \\ Lexington, MA 02421, USA \\ ${ }^{2}$ Basel Pharmacoepidemiology Unit, Division of Clinical Pharmacology and Toxicology, University Hospital, \\ Spitalsstrasse 26, 4031 Basel, Switzerland \\ ${ }^{3}$ Castlemilk Group Practice, Dougrie Drive, Glasgow G45 9AW, UK
}

Correspondence should be addressed to Hershel Jick, hjick@bu.edu

Received 4 May 2011; Accepted 16 June 2011

Academic Editor: Huibert Burger

Copyright (C) 2011 Hershel Jick et al. This is an open access article distributed under the Creative Commons Attribution License, which permits unrestricted use, distribution, and reproduction in any medium, provided the original work is properly cited.

Background. Initially the course of the 2009 swine flu pandemic was uncertain and impossible to predict with any confidence. An effective prospective data resource exists in the United Kingdom (UK) that could have been utilized to describe the scope and extent of the swine flu outbreak as it unfolded. We describe the 2009 swine flu outbreak in the UK as recorded daily by general practitioners and the potential use of this database for real-time tracking of flu outbreaks. Methods. Using the General Practice Research Database, a real-time general practice, electronic database, we estimated influenza incidence from July 1998 to September 2009 according to age, region, and calendar time. Results. From 1998 to2008, influenza outbreaks regularly occurred yearly from October to March, but did not typically occur from April to September until the swine flu outbreak began in April 2009. The weekly incidence rose gradually, peaking at the end of July, and the outbreak had largely dissipated by early September. Conclusions. The UK swine flu outbreak, recorded in real time by a large group of general practitioners, was mild and limited in time. Simultaneous online access seemed feasible and could have provided additional clinical-based evidence at an early planning stage of the outbreak.

\section{Introduction}

An outbreak of influenza in Mexico in April 2009 was found to be due to a new H1N1 strain of influenza A (subsequently referred to as "swine flu"). A worldwide spread of the virus rapidly ensued. Initially, the nature and scope of a swine flu pandemic was uncertain and, in view of the high variability of influenza outbreaks in the past 40 years, it was impossible to predict with any confidence [15]. In the UK and elsewhere, influenza surveillance systems have operated for decades to follow the course of such outbreaks, and subsequent to the initial swine flu outbreak additional surveillance efforts were instituted in the UK (http://www.hpa.org.uk/).

In the June 20, 2009 issue of The Lancet, Tran and colleagues commended the UK efforts to address the outbreak, but noted that "our major concern about the current response to $\mathrm{H} 1 \mathrm{~N} 1$ has been the dearth of any systematic, prospective, patient-oriented clinical research alongside the commendable public health response to the pandemic" [6]. In fact, an effective, systematic, prospectively designed data resource did exist in the UK at the time and could have been used to describe course of the outbreak in the general population, as diagnosed by a representative sample of some 1000 general practitioners on a daily basis, from the start as it unfolded.

The United Kingdom (UK) General Practice Research Database (GPRD) is a computer-based electronic medical record system that was established in 1990 to replace the traditional paper medical record. It provides a comprehensive, chronological, validated record of diagnoses, vaccinations, prescribed medicines, and other relevant clinical information recorded during the consultation with the patient [ 7 , 8]. This resource has been used to describe in detail the 
incidence of mumps, measles, and influenza in the UK in relation to age, calendar time, geographic location, and prior vaccination as recorded electronically in real time by some 350 UK general practices located from Northern Ireland to Southeast England [5, 9, 10]. In this paper, we describe in detail the first wave of the swine flu outbreak in the UK in the spring and summer of 2009.

\section{Methods}

2.1. Data Source. The contributing UK general practices have provided continuous standardized, computer-recorded, anonymous medical information to the Database since 1984. A user group was formed to support the GPs who use the office computers (http://www.nvug.org/) About 3 million people, an estimated 5\% of the UK population, are registered in these general practices $[9,11]$. Initially, the data collection was a collaborative effort with Vamp Health, the private business that produced the general practice office computers [12]. The data was organized and formatted into a file structure that provides rapid and efficient access for observational research [7]. In the late 1990s, the responsibility for data collection was transferred to the Medicines and Healthcare products Regulatory Agency (MHRA).

The Database closely reflects the age, gender, and geographic distribution of the UK and has remained stable in size over the last 20 years [11]. Some 1,000 general practitioners (GPs) who contribute to the database use office computers with software specifically designed by an experienced GP for real-time entry and have agreed to provide the information for research purposes [7, 12, 13]. The GPs were initially trained to record the medical information on demographics, medical diagnoses, medicines, and vaccines in a standard format using the identical software. Diagnoses and administrative events were recorded based on Oxford Medical Information System (OXMIS) or more recently using Read codes. The patient information is routinely recorded at the time of an office visit. Letters from hospitals and consultants as well as lab results are recorded by GPs as they are received. Most external information relevant to this study came from out-of-hours services and Accident and Emergency Department letters that were usually received and recorded the next day. The comprehensive nature of the information on clinical diagnoses, vaccines, and drug exposure recorded in the database has been extensively validated and found to be of high quality for the purpose of conducting epidemiological research [12-14]. Numerous studies based on this Database have addressed the safety and effectiveness of vaccines $[9,10,15,16]$.

The protocol was reviewed and approved by the Independent Scientific Advisory Committee (ISAC) of the MHRA.

\subsection{Case Identification and Statistical Analyses. We derived} the monthly number of GP-recorded cases of influenza for the time period from June 1998 through September 2009. We compared the number of cases recorded in the warm weather months (April through September) of 2009 during the swine flu outbreak with those previously recorded since



FIGURE 1: Monthly number of influenza cases for June 1998 through September 2009. Seasonal activity labels from HPA Centre for Infections [17].

1998 in relation to age and season. We also derived the cumulative six-month incidence according to age $(<5,5-$ 9, 10-19, 20-49, 50-69, 70+) and season (cold weather months October-March and warm weather months AprilSeptember) and separately a weekly incidence for the period from April through September 2009 according to age and region. Incidence rates are calculated by identifying the number of GP-diagnosed cases of influenza and dividing by the number people actively enrolled in the database according to age and geography for each relevant calendar time period.

We provide the related mortality, pregnancy status, and frequency of prescribing the antiviral drugs oseltamivir (Tamiflu) and zanamivir (Relenza) in those with a diagnosis of influenza recorded in their electronic record. Finally, we report on related telephone encounters to the GP within 48 hours of the recorded influenza diagnosis.

\section{Results}

The monthly number of influenza cases diagnosed and recorded in the Database from June 1998 to September 2009 is shown in Figure 1. Higher than average monthly outbreaks, that reached a peak in the month of January, occurred in the traditional cold weather months (OctoberMarch) of 1998-1999 and 1999-2000. In the period of October 1999-March 2000, 10,528 cases of influenza were recorded in the month of January 2000, the largest monthly outbreak of influenza in more than 10 years.

In 2001, the UK government set targets for influenza vaccination to be increased in the elderly and people at high risk, which resulted in a substantially increased frequency of vaccination in the following years [5]. During the period from 2001 through March 2009, the peak monthly incidence of influenza during the cold weather months fell to below average levels (see Figure 1). In the warm weather months, the monthly incidence of diagnosed influenza was low for the period of 1998 through 2008, regularly fewer than 600 cases per month.

The total number of influenza cases recorded in the cold weather months (October-March) and separately in the warmer months (April-September) is shown in Figure 2 


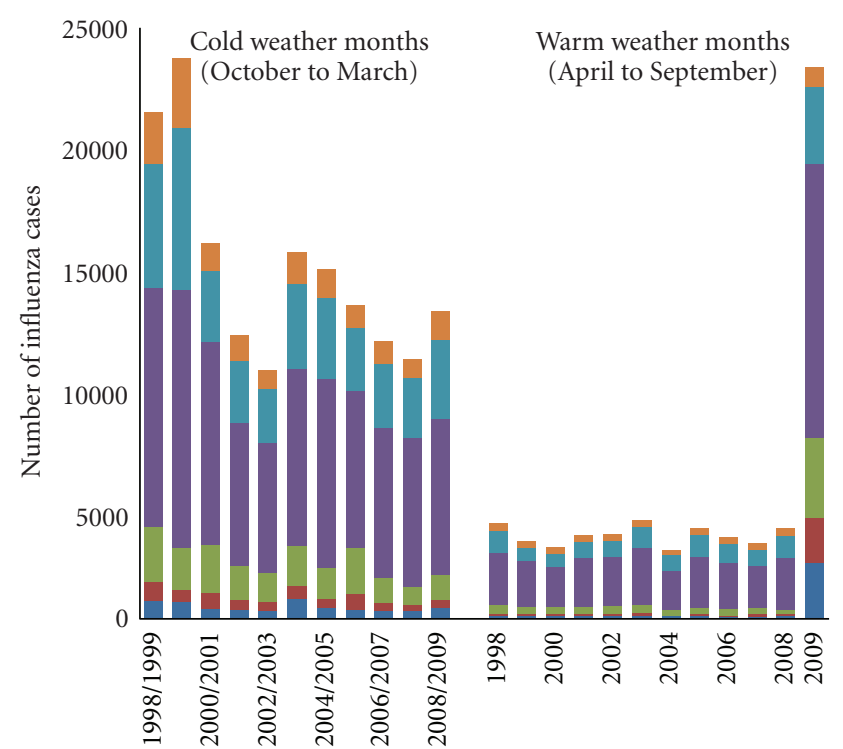

Age groups

- $70+$ years

- 50-69 years

- 20-49 years

- 10-19 years

- 5-9 years

- $<5$ years

Figure 2: Number of influenza cases by age group for cold and warm weather months for 1998-2009.

according to age categories. In the cold weather months, more than $70 \%$ of cases were regularly diagnosed in people aged 20-69. Influenza was infrequently diagnosed in the warm weather months at any age category from 1998 through 2008. Only 606 cases were diagnosed by the GPs in April 2009.

The number of recorded cases of influenza began to rise towards the end of May 2009 (1,068 cases) and a warm weather month outbreak, not seen in the UK for many decades, ensued (Figure 2). The number of cases rose modestly in June ( 1,898 cases) but increased sharply in July to 15,070 cases, the highest monthly number of influenza cases recorded in the Database at any time. The number of cases declined in August (3,065 cases) and fell further in September (1,538 cases), and it was clear that the swine flu outbreak was rapidly dissipating. By the end of September, the outbreak was almost over and in early February 2010, the NHS announced that the pandemic flu phone lines would be closed, a sign that the outbreak in Britain was largely over [18].

The weekly number of cases of influenza recorded in real time before, during, and after the outbreak of 2009, according to age, is shown in Figure 3. Examination of the findings according to individual general practices reveals that the swine flu outbreak was first recorded in a single practice in Glasgow in the 25th week of 2009 (7-13 June). It became fully evident in the 26th and 27th weeks (1420 and 21-27 June) of 2009 when 356 cases were recorded, disproportionately in young children. It rapidly reached a peak in the 29th and 30th weeks (12-18 and 19-25 July) when more than 5,000 cases were recorded in each week. By

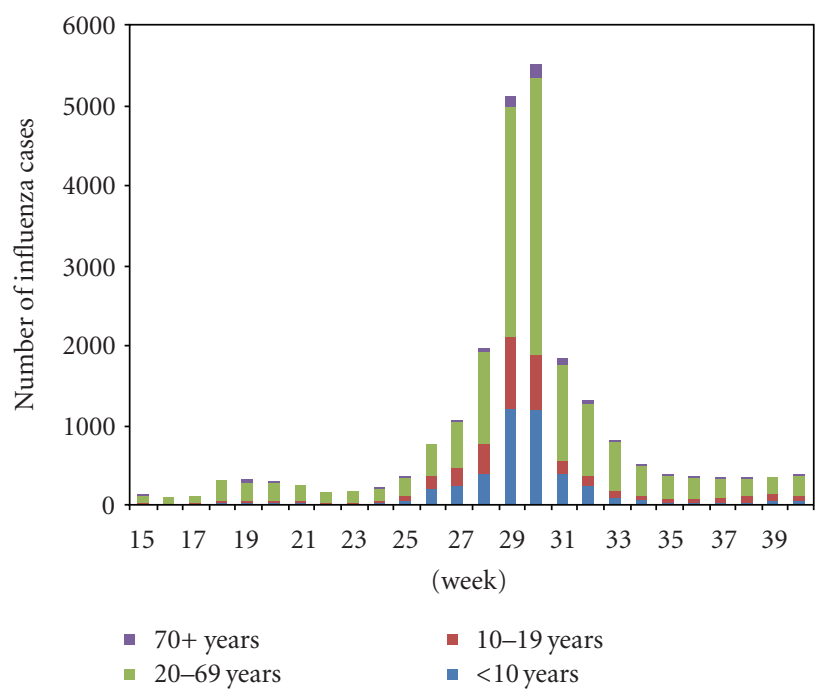

Figure 3: Number of influenza cases by week for the period of April through September 2009 by age.

the end of the 32nd week (2-8 August) of 2009, it had fallen to 1,314 cases only two weeks after the peak. It had fallen further to 338 cases in the third week of September.

We estimated six-month cumulative incidence rates (IRs), according to age, from April through September 2009 by dividing the number of cases in each age category by the total number of people that were at risk for flu in the same age category (i.e., active in the practices during that time). Prior to May 2009, the estimated six-month cumulative incidence rate in all warm weather months (April-September) was regularly less than 1 per 10,000 in children less than 5 years of age. During the swine flu outbreak in the warm weather months of 2009, we estimate that the six month cumulative IR in children less than 5 years of age was about 105 per 10,000, more than 100 times higher than the IR in any warm weather season of the prior 10 years for this age group. The IR was also more than 5 times higher than those recorded in any cold weather month.

During the swine flu outbreak, the six-month cumulative incidence estimate for those age 70 years or older was about 10 per 10,000, a rate similar to those recorded in most prior cold weather seasons in this age group, but only about one tenth the IR in children aged less than 5 years.

Estimates of the overall weekly incidence rates (IRs) according to region are shown in Figure 4 for June through September 2009. After modest increases occurred in the Midlands, London and Scotland in mid to late June, incidence rates rose dramatically in all areas of the UK in the 29 and 30th weeks of 2009. The highest rates were recorded in the Southwest (primarily Gloucester) and South (primarily London). The incidence rates fell precipitously in early August in all regions and had dissipated by the end of September except for a few practices in the East of Scotland.

In the 29th week of 2009 at the peak of the outbreak, the estimated weekly incidence rate was highest in children less than 5 years of age $(44.4$ per 10,000$)$. It fell progressively with 


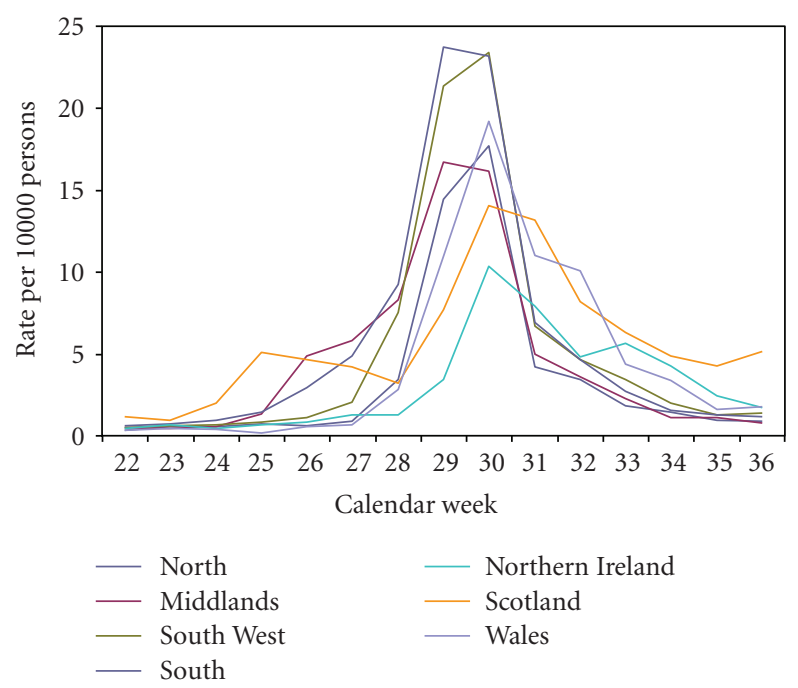

FIgURE 4: Weekly rate of swine flu from 2009 calendar week 22 through week 36 by region.

increasing age to 3.4 per 10,000 in adults 70 years of age or older.

Weekly incidence rates for all ages combined in major city postal areas where the largest outbreaks occurred are shown in Figure 5. Incidence rose from low background levels to a level of 12.7 per 10,000 in Glasgow in week 25 (7-13 June). A major outbreak occurred in Birmingham in week 26 (14-20 June) when the incidence rate reached 25.8 per 10,000 . Rates began to rise substantially in the London area in weeks 26 and 27 and continued at relatively modest levels until week 31 . The largest outbreak $(43.8$ per 10,000$)$ in the UK arrived in Gloucester in week 29 (12-18 July) and continued at this level through week 30 . Incidence rates in Manchester, Cardiff/Newport, Belfast, and all other postal code areas combined were materially below weekly levels attained in the four largest city areas. The swine flu incidence rate remarkably fell to levels at or below 10 per 10,000 in week 32 in all areas.

3.1. Related Findings. In the large cold weather outbreak of 1999-2000, which occurred primarily in middle age and elderly people, 71 people died within 30 days of the diagnosis of influenza. Over 80 percent of those who died were older than 70 years of age. During the swine flu outbreak of 2009, which occurred primarily in children, only 17 cases were recorded as having died within 30 days of the influenza diagnosis. Nine were less than age of 70 years old, all of whom were older than age 30 . Six of these had prior chronic illnesses that predispose to influenza and its complications [4]. None of those who died were recorded as being pregnant.

During July 2009, the National Pandemic Flu Service was established and patients were encouraged to contact this service by telephone. They were assessed according to an algorithm, and if influenza was diagnosed then a prescription for oseltamivir or zanamivir was issued. The extent to which this information was fully recorded in the patient record is uncertain. As this service only applied to England, we have compared data from the other three UK countries and can

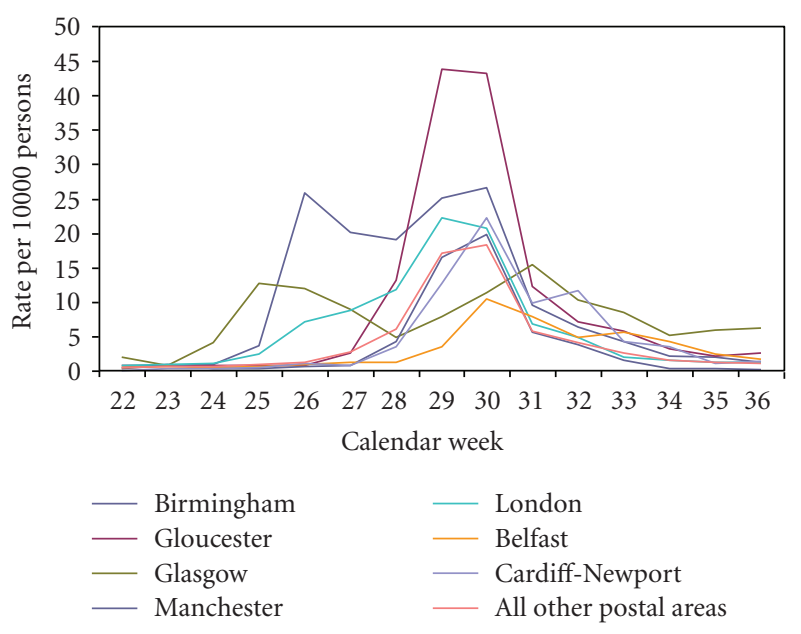

FIGURE 5: Weekly rate of swine flu from 2009 calendar from weeks 22 through 36 by postal area.

find no evidence that the introduction of this service affected data recording.

The number of people prescribed oseltamivir (Tamiflu) in April 2009 was 63. This number rose to 11,379 in July, but fell to 324 in September as the swine flu outbreak dissipated. Only 367 people were prescribed zanamivir (Relenza) during the entire 2009 flu outbreak, more than 90\% of whom were females aged 10-49 recorded as being pregnant at the time. Initially, oseltamivir was prescribed to cases and contacts in an attempt to control the spread of the infection. Subsequently, this policy was changed and only cases were given treatment. Zanamivir was prescribed to patients who were pregnant or unable to take oseltamivir.

For the period from July 1998 through March 2009, less than $0.1 \%$ of those who were diagnosed with influenza had an associated prior telephone call to the GP recorded in the Database within 48 hours of the date of influenza diagnosis. During July 2009, after the government recommended that people with symptoms of influenza report it by phone rather than visit their GP to avoid unnecessary spread of the virus, the GPs contemporaneously entered a "telephone encounter" into the record in about $25 \%$ of those diagnosed with influenza in July and August.

We identified 7,332 women aged 15-44 who were diagnosed with influenza ("cases") during the swine flu outbreak. Of these, $501(6.8 \%)$ were recorded as being pregnant at the time of the influenza diagnosis. For comparison, we identified 29,318 control women who did not have diagnosed influenza, matched $4: 1$ to cases on year of birth, general practice, and the date of diagnosis in the case. Among controls, 1,641 (5.6\%) were recorded as being pregnant.

Vaccination against seasonal influenza has been regularly administered in October, November, and December in the UK in the past decade, particularly in the elderly and those at high risk for complications. Influenza vaccination in the warm weather months has been rare [5]. In the four months prior to the swine flu outbreak, less than $0.5 \%$ of people of any age were vaccinated with any influenza vaccine strain. 
Recent influenza vaccination played little if any role in the swine flu outbreak of 2009.

\section{Discussion}

By late 2009, the extent and severity of the swine flu outbreak in the UK had been convincingly reported publicly as being of short duration and relatively mild [19]. Subsequently, in early April 2010, the government announced that 60 million doses of swine flu vaccine had been cancelled [20]. On June 4, 2010, the Associated Press reported that the Council of Europe had accused the World Health Organization and European governments of vastly exaggerating the health risks of swine flu [21].

The results reported in this paper provide populationbased, real-time GP entry of influenza diagnoses, presumed to be primarily swine flu, from May to September 2009, that are consistent with the published reports $[19,22]$. Since the Database represents about $5 \%$ of the UK population, we estimate that the number of GP-diagnosed cases of swine flu in the UK was in the range of 500,000 people during May through September 2009. During the month of July 2009 alone, the number of people recorded by GPs as having influenza in the UK is estimated to have been about 300,000.

Our results add additional quantitative detail on the age, regional distribution, and course of the swine flu outbreak. They confirm that, unlike prior influenza outbreaks, the swine flu primarily affected young children $[19,22]$. All regions of the UK were affected. Incidence rates consistently rose slowly in late May and June, peaked in July, and unexpectedly fell rapidly and progressively in August and September in all regions.

Most importantly, we document that a daily account of the swine flu experience in the UK was being recorded by GPs in real time. This information, in addition to the other surveillance systems used, could have been utilized at little cost to help inform the government's public health response to this outbreak. As the GPRD data is comprised of validated clinical diagnoses, vaccinations, prescribed medicines, and other relevant clinical information, using the GPRD as an additional surveillance system could help identify populations at the highest risk for infection, as well as to provide insight into the clinical details and outcomes in those infected.

In 2005, we conducted preliminary evaluation of the feasibility of an online, real-time, daily transmission of information on newly marketed drug use and outbreaks of viral infections to a central repository of the Database that was already in place and designed to receive, rapidly process, and access this type of additional information. Instant transmission of GP-recorded diagnoses of flu to a central repository appeared to be feasible at a negligible cost. Our submission to a government agency to proceed with the project was not approved and it was not followed up. Had this unique online data collection system been implemented successfully, the daily mapping of the swine flu outbreak in the UK could have been accomplished in a large defined UK population. This information could have been vital for WHO and countries around the world that were considering how to respond to the impending swine flu pandemic.

It has been reported that there was a second wave of the 2009 swine flu that occurred after September [23]. We were unable to evaluate the presence of this with the data currently available to us (through September 2009), although we have no reason to suspect that the continuously recorded data contained in the GPRD would fail to show a second wave. Additionally, it has been reported that there were changes in reporting behavior of the public as the outbreak went on in response to media coverage and the introduction of the National Pandemic Flu Service, which is thought to have directed patients away from GP offices and, thus, GP reporting systems to avoid unnecessary spread of the virus. Our data indicates that many GPs continued to receive reports of influenza over the phone, contemporaneously recording a "telephone encounter" along with the code for influenza in about $25 \%$ of those patients diagnosed with influenza in July and August, and thus not completely eliminating GP recording of the swine flu outbreak. As the data collected by any one surveillance system can be influenced by government and media activities designed to protect public health, there is a need for numerous and varied data sources to track outbreaks, replicate estimates of disease incidence, and provide additional clinical details concerning the population infected.

\section{Conclusions}

The GPs who contribute to the this database continue to record up to date medical information on their patients that can be used to track the incidence of infectious diseases in real time. The GPRD, which is rich in clinical detail, could be used as an additional rapid surveillance data source to assist public health responses to these types of outbreaks.

\section{Conflict of Interests}

The authors declare that they have no competing interests.

\section{Acknowledgment}

The authors are grateful for the excellent contributions made by the general practitioners who continue to provide the comprehensive clinical information on their patients to the GPRD for the past 20 years.

\section{References}

[1] The Lancet Editorial Staff, "Swine influenza: how much of a global threat?” The Lancet, vol. 373, no. 9674, p. 1495, 2009.

[2] R. Coker, "Swine flu: fragile health systems will make surveillance and mitigation a challenge," British Medical Journal, vol. 338, pp. 1087-1088, 2009.

[3] D. M. Fleming and A. J. Elliot, "Lessons from 40 years' surveillance of influenza in England and Wales," Epidemiology and Infection, vol. 136, no. 7, pp. 866-875, 2008.

[4] C. R. Meier, P. N. Napalkov, Y. Wegmüller, T. Jefferson, and H. Jick, "Population-based study on incidence, risk factors, 
clinical complications and drug utilization associated with influenza in the United Kingdom," European Journal of Clinical Microbiology \& Infectious Diseases, vol. 19, pp. 834-942, 2000.

[5] H. Jick, D. P. Chamberlin, and K. W. Hagberg, "Vaccine policy and the incidence of influenza," Epidemiology, vol. 21, no. 2, pp. 270-271, 2010.

[6] T. H. Tran, G. M. Ruiz-Palacios, F. G. Hayden, and J. Farrar, "Patient-oriented pandemic influenza research," The Lancet, vol. 373, no. 9681, pp. 2085-2086, 2009.

[7] H. Jick, "The general practice research database," in Side Effects of Drugs, Annual 27, J. K. Aronson, Ed., pp. xxxi-xxxiii, Elsevier, Amsterdam, The Netherlands, 2004.

[8] H. Jick, "A database worth saving," The Lancet, vol. 350, no. 9084, pp. 1045-1046, 1997.

[9] H. Jick, D. P. Chamberlin, and K. W. Hagberg, "The origin and spread of a mumps epidemic: United Kingdom 2003-2006," Epidemiology, vol. 20, no. 5, pp. 656-661, 2009.

[10] H. Jick and K. W. Hagberg, "Measles in the United Kingdom 1990-2008 and the effectiveness of measles vaccines," Vaccine, vol. 28, no. 29, pp. 4588-4592, 2010.

[11] J. Hollowell, "General practice research database (GPRD): scope and quality of data," Office of Population Censuses and Statistics (OPCS, now known as Office of National Statistics [ONS]), pp. 1-20, 1994.

[12] H. Jick, S. S. Jick, and L. E. Derby, "Validation of information recorded on general practitioner based computerized data resource in the United Kingdom," British Medical Journal, vol. 302, no. 6779, pp. 766-768, 1991.

[13] H. Jick, "Learning how to control biases in studies to identify adverse effects of drugs: a personal history," Journal of the Royal Society of Medicine, vol. 102, no. 4, pp. 160-164, 2009.

[14] S. S. Jick, J. A. Kaye, C. Vasilakis-Scaramozza et al., "Validity of the general practice research database," Pharmacotherapy, vol. 23, no. 5, pp. 686-689, 2003.

[15] J. A. Kaye, M. M. Mar Melero-Montes, and H. Jick, "Mumps, measles, and rubella vaccine and the incidence of autism recorded by general practitioners: a time-trend analysis," British Medical Journal, vol. 322, no. 7284, pp. 460-463, 2001.

[16] H. Jick, C. Vasilakis-Scaramozza, and S. S. Jick, "Live attenuated polio vaccine and the risk of intussusception," British Journal of Clinical Pharmacology, vol. 52, no. 4, pp. 451-453, 2001.

[17] Influenza/Respiratory Virus Team and HPA Centre for Infections, "HPA national influenza season summary 2007/08," Report for 5 August 2008.

[18] R. Smith, "NHS flu line being switched off in sign outbreak is over," The Telegraph, 2010, http://www.telegraph.co.uk/ health/swine-flu/7145592/NHS-flu-line-being-switched-offin-sign-outbreak-is-over.html.

[19] L. J. Donaldson, P. D. Rutter, B. M. Ellis et al., "Mortality from pandemic A/H1N1 2009 influenza in England: public health surveillance study," British Medical Journal, vol. 339, p. b5213, 2009.

[20] S. Mayor, "Department of health agrees deal with GSK to cancel part of swine flu vaccine order," British Medical Journal, vol. 340, p. c1946, 2010.

[21] The Associated Press, "Council of Europe slams WHO handling of swine flu," New York Times, 2010.

[22] Health Protection Agency, "Pandemic (H1N1) 2009 Influenza: UK situation at 20 August 2009," Health Protection Report 2009 3(33).

[23] Influenza/Respiratory Virus Team and HPA Centre for Infections, "Epidemiological report of pandemic (H1N1) 2009 in the UK,” April 2009-May 2010. 


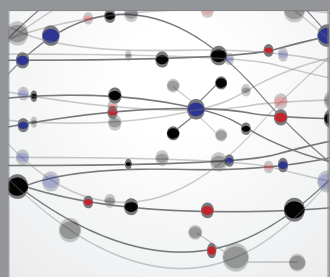

The Scientific World Journal
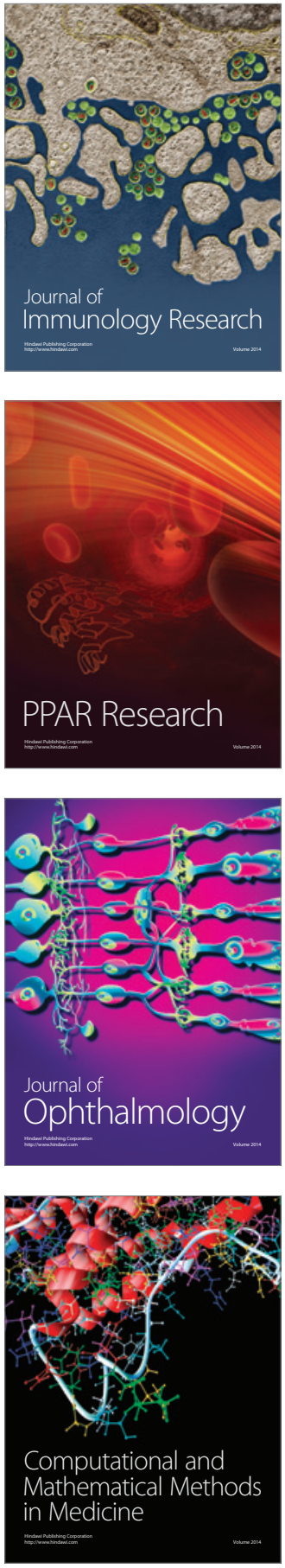

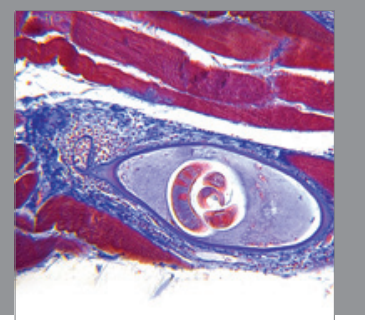

Gastroenterology

Research and Practice


\section{Hindawi}

Submit your manuscripts at

http://www.hindawi.com




Journal of
Diabetes Research

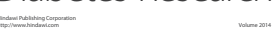



Disease Markers
